Publ. Math. Debrecen

84/1-2 (2014), 259-277

DOI: $10.5486 /$ PMD.2014.5956

\title{
Equal values of standard counting polynomials
}

\author{
By KÁLMÁN GYŐRY (Debrecen), TÜNDE KOVÁCS (Debrecen),
} GYÖNGYVÉR PÉTER (Debrecen) and ÁKOS PINTÉR (Debrecen)

Dedicated to Professor Lajos Tamássy on his 90th birthday

\begin{abstract}
The following discrete geometrical question provides a background for some classical diophantine problems. For given positive integers $m, n$, can an $m$ dimensional and an $n$-dimensional unit cube, simplex, pyramid or octahedron contain equally many integral points? Apart from some trivial cases, the question leads to 9 families of diophantine equations, see Table 1 . In this paper we give a brief survey of known results on these equations, and prove some new theorems concerning the solutions.
\end{abstract}

\section{Introduction}

The most fundamental polynomials counting integer points are $X^{n}$ in an $n$-dimensional unit cube, $\left(\begin{array}{c}X+n \\ n\end{array}\right)$ in a standard $n$-simplex,

$$
S_{n-1}(X)=1^{n-1}+2^{n-1}+\ldots+X^{n-1}
$$

in an $n$-dimensional pyramid, and

$$
P_{n}(X)=\sum_{j=0}^{n}\left(\begin{array}{l}
n \\
j
\end{array}\right)\left(\begin{array}{c}
X+n-j \\
n
\end{array}\right)
$$

Mathematics Subject Classification: 11D41.

Key words and phrases: diophantine equations, counting polynomials.

Research was supported in part by the Hungarian Academy of Sciences, OTKA grants T67580, K75566, K100339, NK101680, NK104208 and by the European Union and the European Social Fund through project Supercomputer, the national virtual lab (grant no.: TAMOP-4.2.2.C11/1/KONV-2012-0010). 
for octahedron in dimension $n$, see [4, Chapter 2]. The purpose of this paper is to consider the possible equal values of these polynomials in case of integral variables. In other words, for given positive integers $m, n$, how often can two bodies (unit cube, simplex, pyramid, octahedron) of dimensions $m$ and $n$, respectively, contain equally many integral points? It is a bit surprising that this discrete geometrical question is the common background of some classical diophantine problems. One can see that the above problems lead to 9 nontrivial families of diophantine equations, see Table 1 . We give a survey of known results concerning these equations. Further, we prove some new theorems for the solutions. For each family of solutions, the following three types of results can be established. An ineffective finiteness theorem for the general case obtained by Bilu-Tichy Theorem, an effective result based on Baker's theory when one of the dimensions involved is small, and the resolution by computer algebraic packages if both dimensions are small.

\begin{tabular}{|c|c|c|}
\hline No & Equation & Remark \\
\hline 1 & $S_{m}(x)=S_{n}(y)$ & $n>m \geq 1$ \\
\hline 2 & $S_{m}(x)=y^{n}$ & $m \geq 1, n \geq 2,(m, n) \notin\{(1,2),(3,2),(3,4),(5,2)\}$ \\
\hline 3 & $S_{m}(x)=\left(\begin{array}{c}y \\
n\end{array}\right)$ & $m \geq 1, n \geq 2,(m, n) \neq(1,2)$ \\
\hline 4 & $S_{m}(x)=P_{n}(y)$ & $m \geq 1, n \geq 2,(m, n) \neq(1,2)$ \\
\hline 5 & $\left(\begin{array}{l}x \\
m\end{array}\right)=y^{n}$ & $m \geq 2, n \geq 2,(m, n) \neq(2,2)$ \\
\hline 6 & $\left(\begin{array}{c}x \\
m\end{array}\right)=\left(\begin{array}{c}y \\
n\end{array}\right)$ & $n>m \geq 2$ \\
\hline 7 & $\left(\begin{array}{c}x \\
m\end{array}\right)=P_{n}(y)$ & $m \geq 2, n \geq 2,(m, n) \neq(2,2)$ \\
\hline 8 & $P_{m}(x)=y^{n}$ & $m \geq 2, n \geq 2,(m, n) \neq(2,2)$ \\
\hline 9 & $P_{m}(x)=P_{n}(y)$ & $n>m \geq 2$ \\
\hline
\end{tabular}

Table 1: The investigated families of diophantine equations

\section{Lemmas and auxiliary results}

First we note that $S_{n-1}(X)$ can be expressed in the form

$$
S_{n-1}(X)=\frac{1}{n}\left(B_{n}(X+1)-B_{n}(0)\right),
$$

where $B_{n}(X)$ denotes the $n$-th Bernoulli polynomial which is of degree $n$ and has its coefficients in $\mathbb{Q}$. 
We now collect some lemmas to prove our new results. The first one deals with the simple zeros of a family of polynomials. Let $n$ be a positive integer, $f(X)$ an integer-valued polynomial with $\operatorname{deg} f(X) \leq n-1$, and $g(X)$ a polynomial with rational integer coefficients.

Lemma 1. Suppose that $n \geq 6$ and let $p$ denote a prime for which

$$
\frac{2}{3} n<p \leq n \text {. }
$$

If $a_{n}$ is an integer not divisible by $p$ then the polynomial

$$
F(X)=a_{n}\left(\begin{array}{l}
X \\
n
\end{array}\right)+f(X)+g(X)
$$

has at least $\left[\frac{n}{3}\right]+1$ simple zeros.

Proof. This is the Theorem in [41].

The following result provides an effective upper bound for the solutions to the hyperelliptic equations.

Lemma 2. Let $f$ be a polynomial with rational coefficients and suppose that it possesses at least three simple zeros. Then the equation $f(x)=y^{2}$ in unknown integers $x, y$ implies $\max (|x|,|y|)<c_{1}$, where $c_{1}$ is an effectively computable constant depending on the degree and the maximum height of the coefficients of $f$.

Proof. See [3].

There is a similar result for superelliptic equations.

Lemma 3. Let $f$ be a polynomial with rational coefficients and suppose that it possesses at least two simple zeros. Then the equation $f(x)=y^{k}$ in unknown integers $x, y, k \geq 2$ implies $\max (|x|,|y|, k)<c_{2}$, where $c_{2}$ is an effectively computable constant depending on the degree and the maximum height of the coefficients of the polynomial $f$.

Proof. For the bound on $k$, see [50], and on $|x|,|y|$ see [3] .

The next results are used in the proofs of our effective statements.

Lemma 4. Let $m>1, r, s \neq 0$ be fixed integers. Then apart from the cases when $m=3, r=0$ or $s+64 r=0 ; m=5, r=0$ or $s-324 r=0$, the equation

$$
s\left(1^{m}+2^{m}+\ldots+x^{m}\right)+r=y^{n}
$$

in integers $x>0, y$ with $|y| \geq 2$, and $n \geq 2$ has only finitely many solutions which can be effectively determined. 
Proof. This is Theorem 2.2 in [47].

Lemma 5. Let $a, b, c$ and $m$ be given integers with $a b \neq 0$ and $m \geq 3$. Apart from the cases when $m=4, c / a=-1 / 24$ or $3 / 128, n=2$ and $b / a$ is not a square, the diophantine equation

$$
a\left(\begin{array}{c}
x \\
m
\end{array}\right)=b y^{n}+c
$$

has only finitely many solutions in $x, y>1, n \geq 2$ and all these solutions can be effectively bounded in terms of $a, b, c$ and $m$.

Proof. This is the main result of [58].

Lemma 6. Let $a, b, m, n$ be integers with $a \neq 0, m \geq, n>2$. The equation

$$
S_{m}(x)=a\left(\begin{array}{l}
y \\
n
\end{array}\right)+b
$$

in integers $x$ and $y$ has only finitely many solutions apart from the following possible exceptions

$$
(m, n) \in\{(1,4),(2,3),(3,4)\} .
$$

Proof. This is a special case of Theorem 2 in [46].

We will introduce some notation to recall the finiteness criterion by Bilu and Tichy. In what follows $\alpha$ and $\beta$ are nonzero rational numbers, $\mu, \nu$ and $q$ are positive integers, $p$ is a nonnegative integer and $\nu(X) \in \mathbb{Q}[X]$ is a nonzero polynomial (which may be constant).

A standard pair of the first kind is $\left(X^{q}, \alpha X^{p} \nu(X)^{q}\right)$ or switched, $\left(\alpha X^{p} \nu(X)^{q}, X^{q}\right)$, where $0 \leq p<q,(p, q)=1$ and $p+\operatorname{deg} \nu(X)>0$.

A standard pair of the second kind is $\left(X^{2},\left(\alpha X^{2}+\beta\right) \nu(X)^{2}\right)$ (or switched).

Denote by $D_{\mu}(X, \delta)$ the $\mu$ th Dickson polynomial, defined by the functional equation $D_{\mu}(z+\delta / z, \delta)=z^{\mu}+(\delta / z)^{\mu}$. As it is well-known, we have the explicit formula

$$
D_{\mu}(X, \delta)=\sum_{i=0}^{[\mu / 2]} d_{\mu, i} X^{\mu-2 i},
$$

with

$$
d_{\mu, i}=\frac{\mu}{\mu-i}\left(\begin{array}{c}
\mu-i \\
i
\end{array}\right)(-\delta)^{i} .
$$

A standard pair of the third kind is $\left(D_{\mu}\left(X, \alpha^{\nu}\right), D_{\nu}\left(X, \alpha^{\mu}\right)\right)$, where $\operatorname{gcd}(\mu, \nu)=1$.

A standard pair of the fourth kind is $\left(\alpha^{-\mu / 2} D_{\mu}(X, \alpha),-\beta^{-\nu / 2} D_{\nu}(X, \beta)\right)$, where $\operatorname{gcd}(\mu, \nu)=2$.

A standard pair of the fifth kind is $\left(\left(\alpha X^{2}-1\right)^{3}, 3 X^{4}-4 X^{3}\right)$ (or switched). 
Lemma 7. Let $R(X), S(X)$ be nonconstant polynomials such that the equation $R(x)=S(y)$ has infinitely many solutions in rational integers $x, y$. Then $R(X)=\phi(f(\kappa(X)))$ and $S(X)=\phi(g(\lambda(X)))$ where $\kappa(X), \lambda(X) \in \mathbb{Q}[X]$ are linear polynomials, $\phi(X) \in \mathbb{Q}[X]$, and

is a standard pair.

$$
(f(X), g(X))
$$

Proof. This is a consequence of the main result of [10].

The next result will be useful for the application of the previous lemma (cf. [51] and [44]).

Lemma 8. The product of two or more consecutive positive integers is never a perfect power.

Proof. For the proof we refer to [23].

We need the following technical lemma. Let $a, b, \tilde{a}, \tilde{b}, \bar{a}, \bar{b}$ be rational numbers with $a \tilde{a} \bar{a} \neq 0$.

Lemma 9. None of the polynomials $\left(\begin{array}{c}a X+b \\ m\end{array}\right)$ and $P_{m}(\tilde{a} X+\tilde{b})$ is of the form $e_{1} X^{m}+e_{0}$ with $e_{1} \in \mathbb{Q} \backslash\{0\}$ and $m \geq 3$ or $e_{1} D_{m}(X, \alpha)+e_{0}$ with $e_{1}, \alpha \in \mathbb{Q} \backslash\{0\}$ and $m \geq 5$. The polynomial $S_{m}(\bar{a} X+\bar{b})$ is not of the form $e_{1} X^{q}+e_{0}$ with $q \geq 3$ or $e_{1} D_{\nu}(X, \alpha)+e_{0}$ with $\nu>4$, where $\alpha, e_{1}, e_{0}$ are rational numbers with $e_{1} \neq 0$.

Proof. For the fact that $\left(\begin{array}{c}a X+b \\ m\end{array}\right)$ is not of the form $e_{1} X^{m}+e_{0}$ with $m \geq 3$ we refer to [8, Lemma 5.2].

Now suppose that

$$
\left(\begin{array}{c}
a X+b \\
m
\end{array}\right)=e_{1} D_{m}(X, \alpha)+e_{0}
$$

for an integer $m \geq 5$ and $\alpha \in \mathbb{Q} \backslash\{0\}$ and set

$$
\left(\begin{array}{c}
a X+b \\
m
\end{array}\right)=\sum_{i=0}^{m} c_{i} X^{i}
$$

On comparing the corresponding coefficients, an easy calculation shows that

$$
\begin{gathered}
c_{m}=\frac{a^{m}}{m !}=e_{1}, \\
c_{m-1}=\frac{a^{m-1}\left(b-\frac{m-1}{2}\right)}{(m-1) !}=0,
\end{gathered}
$$




$$
c_{m-2}=\frac{a^{m-2}\left(12 b^{2}+12(1-m) b+3 m^{2}-7 m+2\right)}{24(m-2) !}=-e_{1} \alpha m,
$$

and

$$
\begin{aligned}
c_{m-4} & =\frac{a^{m-4}\left(240 b^{4}+480(1-m) b^{3}+f_{1}(m) b^{2}+f_{2}(m) b+f_{3}(m)\right)}{5760(m-4) !} \\
& =\frac{e_{1} m(m-3) \alpha^{2}}{2},
\end{aligned}
$$

where $f_{1}(m)=120\left(3 m^{2}-7 m+2\right), f_{2}(m)=120\left(-m^{3}+4 m^{2}-3 m\right)$ and $f_{3}(m)=$ $15 m^{4}-90 m^{3}+125 m^{2}-18 m-8$. Using the second equation, we have $b=\frac{m-1}{2}$ and thus

$$
c_{m-2}=-\frac{a^{m-2}(m+1)}{24(m-2) !}=-e_{1} \alpha m
$$

and

$$
c_{m-4}=\frac{a^{m-4}\left(5 m^{2}+12 m+7\right)}{5760(m-4) !}=\frac{e_{1} m(m-3) \alpha^{2}}{2} .
$$

From these relations with $c_{m}=\frac{a^{m}}{m !}=e_{1}$ we get

$$
\frac{(m-1)(m+1)}{24}=a^{2} \alpha
$$

and

$$
\frac{(m+1)(5 m+7)(m-1)(m-2)}{2880}=a^{4} \alpha^{2},
$$

that is

$$
(m+1)(m-1)=\frac{(5 m+7)(m-2)}{5}
$$

and $m=3$, a contradiction.

The proof of the corresponding statements for the polynomials $P_{m}(\tilde{a} X+\tilde{b})$ and $S_{m}(\bar{a} X+\bar{b})$ can be found in [9].

\section{New and known results}

Family 1. Equation

$$
S_{m}(x)=S_{n}(y),
$$

where $n>m \geq 1$ are fixed and $x, y$ are unknown integers. 
For $(m, n)$ with $m=1$ and $m=3$, BRINDZA and PintéR [13] proved some effective finiteness results for the solutions $x$ and $y$. Their proof is based on the structure of zeros of the corresponding shifted Bernoulli polynomials. In the same paper they obtained an ineffective finiteness result for an infinite class of pairs $(m, n)$ using Davenport-Lewis-Schinzel Theorem. Later, applying BiluTichy Theorem, the authors of [8] extended this statement to every pair $(m, n)$. For small values of $m$ and $n$ the problem leads to certain elliptic curves. For the resolution of the special cases $(m, n)=(1,2),(1,3),(1,5),(1,7)$ we refer to [2] and [55], [16] and [38], [33], [37], respectively. We propose the following

Conjecture 1.1. All the solutions to the equation (1.1) in integers $n>m \geq 1$ and $x, y$ are

$$
(m, n, x, y)=(1,2,10,5),(1,2,13,6),(1,3,8,3),(1,5,23,3),(1,5,353,9) .
$$

This conjecture is based upon an extensive numerical investigation. However, its proof seems well beyond the reach of current techniques.

Family 2. Equation

$$
S_{m}(x)=y^{n},
$$

where $m \geq 1, n \geq 2, x \geq 1, y \geq 1$ are unknown integers and $S_{m}(X)=1^{m}+2^{m}+$ $\ldots+X^{m}$.

Equation (2.1) has the solution $(x, y)=(1,1)$ which is called trivial. For $m=n=2,(2.1)$ has only the nontrivial solution $(x, y)=(24,70)$. This was proved by WATSON [57]. In 1956, SChäFfER [49] proved that for fixed $m \geq 1$ and $n \geq 3,(2.1)$ has at most finitely many solutions in $x, y$, unless

$$
(m, n) \in\{(1,2),(3,2),(3,4),(5,2)\},
$$

where in each case, there are infinitely many such solutions.

Schäffer's proof is ineffective. Using Baker's method, GYöRY, TIJDEMAN and Voorhoeve [30] proved a more general and effective result in which the exponent $n$ is also unknown. A special case of their result is the following

Theorem 2.1. For given $m \geq 2$ with $m \notin\{3,5\}$, all solutions $x, y \geq 1, n \geq 2$ of (2.1) satisfy $\max (x, y, n) \leq c_{1}(m)$, where $c_{1}(m)$ is an effectively computable number which depends only on $m$.

Later, Győry, Tijdeman and Voorhoeve [56] showed that for any fixed polynomial $R(X)$ with integral coefficients, the equation

$$
S_{m}(x)+R(x)=y^{n}
$$


has only finitely many solutions in integers $x, y \geq 1, n \geq 2$ provided that $m \geq 2$ is fixed such that $m \neq\{3,5\}$. The proof furnishes an effective upper bound for $n$, but not for $x$ and $y$. An effective version was obtained in a more general form by BRINDZA [11].

PINTÉR [43] proved that for fixed $m>2$, all solutions of (2.1) with $y>1$ satisfy $n<c_{2} m \log m$, where $c_{2}$ is an effectively computable absolute constant.

For fixed $m \geq 2$ with $m \notin\{3,5\}$, Theorem 2.1 makes it possible, at least in principle, to determine all solutions of (2.1). However, the bound $c_{1}(m)$ in Theorem 2.1 is not given explicitly. Moreover, even an explicit value obtained by Baker's method would be too large for practical use. SCHÄFfer [49] was able to prove that for some special pairs $(m, n)$ with small $m, n,(2.1)$ has only the trivial solution. Further, he formulated the following

Conjecture 2.2. For $m \geq 1$ and $n \geq 2$ with $(m, n)$ not in (2.2), equation (2.1) has only one nontrivial solution, namely $(m, n, x, y)=(2,2,24,70)$.

Recently, a considerable progress has been made in this direction. JACOBSON, PINTÉR and WALSH [34] confirmed the conjecture for $n=2$ and for even $m$ with $m \leq 58$. Further, BENNETt, GYŐRY and PINTÉR [6] proved completely Schäffer's conjecture for $m \leq 11$ and for arbitrary $n$.

For fixed $m$ and $(m, n) \neq(3,4)$, BrindzA and Pintér [14] gave the upper bound $\max \left(c_{3}, e^{3 m}\right)$ for the number of solutions of (2.1) with $x, y>1, n>2$, where $c_{3}$ is an effectively computable absolute constant.

In the proofs of the above presented results the first step is to express $S_{m}(X)$ in the form (1). This implies that $S_{m}(X)$ is divisible by $X^{2}(X+1)^{2}$ in $\mathbb{Q}[X]$ if $m>1$ is odd, and by $X(X+1)(2 X+1)$ if $m \geq 2$ is even. Then (2.1) can be reduced both to superelliptic equations and to finitely many binomial Thue equations of the form $A X^{n}-B Y^{n}=1$ in non-zero $X, Y \in \mathbb{Z}$ with fixed non-zero integers $A, B$. Finally, various deep theorems and techniques can be applied to these equations to establish the desired results for equation (2.1).

For more details and related results we refer to the survey paper [29] of GYőRY and PINTÉR.

Family 3. Equation

$$
S_{m}(x)=\left(\begin{array}{c}
y \\
n
\end{array}\right)
$$

where $m \geq 1, n \geq 2$ are fixed integers with $(m, n) \neq(1,2)$ and $x, y$ are unknown integers.

As an easy consequence of Lemma 6 we have 
Theorem 3.1. If $m \geq 1, n \geq 2$ and $(m, n) \neq(1,2)$ then the equation (3.1) has only finitely many solutions in integers $x$ and $y$.

Proof. In view of Lemma 6 we have to check the possible exceptional cases $(m, n) \in\{(1,4),(2,3),(3,4)\}$ only. For $(m, n)=(1,4)$, we get the classical equation

$$
\left(\begin{array}{c}
x+1 \\
2
\end{array}\right)=\left(\begin{array}{l}
y \\
4
\end{array}\right)
$$

and for the resolution of this equation see [19] and [42]. In the case $(m, n)=(2,3)$ we obtain

$$
x(x+1)(2 x+1)=y(y-1)(y-2) .
$$

By using MAPLE one can verify that the genus of the corresponding curve is 1 , so it has only finitely many solutions in integers $x$ and $y$. Finally, if $(m, n)=(3,4)$, our equation takes the form

$$
\left(\frac{x(x+1)}{2}\right)^{2}=\left(\begin{array}{l}
y \\
4
\end{array}\right)
$$

and, by [22], there is no integer solution of this problem.

If $m$ or $n$ is small then we have an effective result.

Theorem 3.2. Let $n \in\{2,4\}$ and $m \geq 1$ with $(m, n) \neq(1,2)$ or $m \in\{1,3\}$ and $n \geq 2$. Then all the solutions of the equation (3.1) in integers $x$ and $y$ are bounded by an effectively computable constant depending only on $m$ or $n$, respectively. Further, if $m=3$ and $n \geq 2$, then there is no solution.

PROOF. In the first case $n=2$ or 4 . Now, our equation (3.1) leads to the equations

or

$$
8 S_{m}(x)+1=(2 y-1)^{2},
$$

$$
24 S_{m}(x)+1=(y(y-3)+1)^{2},
$$

respectively, and Lemma 4 completes the proof. If $m=1$ or $m=3$ we have the equations

$$
(2 x+1)^{2}=8\left(\begin{array}{c}
y \\
n
\end{array}\right)+1,
$$

or

$$
\left(\frac{x(x+1)}{2}\right)^{2}=\left(\begin{array}{l}
y \\
n
\end{array}\right)
$$

respectively. Our statements follow from Lemma 5 and Theorem 5.1 below, respectively. 
Family 4. Equation

$$
S_{m}(x)=P_{n}(y)
$$

where $m \geq 1, n \geq 2$ are fixed integers and $x, y$ are unknown integers.

For small values of $m$ or $n$ we prove the following

Theorem 4.1. If $m \in\{1,3\}$ and $n \geq 2$ or $n \in\{2,4\}$ and $m \geq 1$ then the equation (4.1) implies that $\max (x, y)<c_{1}$, where $c_{1}$ is an effectively computable constant depending only on $n$ or $m$, respectively.

Proof. If $(m, n)=(1,2)$ or $(3,2)$ we have the equations

$$
\left(\begin{array}{l}
x \\
2
\end{array}\right)=2 y^{2}+2 y+1
$$

and

$$
\left(\begin{array}{l}
x \\
2
\end{array}\right)^{2}=2 y^{2}+2 y+1
$$

respectively. One can check that in the first case there is no integer solution in $x$ and $y$, further the second equation represents a genus one curve, so it possesses only finitely many and effectively determinable solutions in $x$ and $y$.

In the sequel we suppose that $m \in\{1,3\}$ and $n \geq 3$. Then we have the following families of equations

$$
(2 x-1)^{2}=8 P_{n}(y)+1
$$

and

$$
\left(\frac{x(x-1)}{2}\right)^{2}=P_{n}(y)
$$

respectively. Since the leading coefficient of the polynomial $P_{n}(X)$ is $\frac{2^{n}}{n !}$, Lemmata 1 and 2 give the proof of our theorem for $n \geq 6$. In the remaining cases a simple calculation shows that the corresponding polynomials have only simple zeros.

Now assume that $n \in\{2,4\}$ and $m \geq 2$. We have the diophantine equations

$$
2 S_{m}(x)=(2 y+1)^{2}
$$

and

$$
3 S_{m}(x)+5=2\left(y^{2}+y+2\right)^{2},
$$

respectively, and Lemma 4 proves the statement of our theorem.

Theorem 4.2. Assume that $m \geq 2, n>2$ and $\operatorname{gcd}(m+1, n)=1$. Then equation (4.1) has only finitely many solutions in integers $x$ and $y$. 
We conjecture that Theorem 4.2 is true omitting the condition for the greatest common divisor of $m+1$ and $n$, cf. [46].

Proof. On supposing the contrary and using Lemma 7 we have

$$
S_{m}(a X+b)=\phi(f(X)), P_{n}(\tilde{a} X+\tilde{b})=\phi(g(X),
$$

where $a, \tilde{a}, b, \tilde{b} \in \mathbb{Q}$ with $a \tilde{a} \neq 0, \phi(X) \in \mathbb{Q}[X]$ and $(f, g)$ is a standard pair. Since the greatest common divisor of $m+1$ and $n$ is 1 , we have that $\operatorname{deg} \phi=1, \phi(X)=$ $e_{0} X+e_{1}$, say, where $e_{0}, e_{1}$ are rational numbers and $e_{0} \neq 0$. Now applying the conditions for $m$ and $n$ we get

$$
\operatorname{deg} f>2, \operatorname{deg} g>2, \operatorname{gcd}(\operatorname{deg} f, \operatorname{deg} g)=1,
$$

and this excludes the standard pairs of the second, fourth and fifth kind. From Lemma 9 we obtain $\max \{m, n\} \leq 5$, and by the conditions for $m, n$ and Theorem 4.1 , the remaining cases are $(m, n)=(2,5),(4,3)$ and $(5,5)$. However, using MAPLE, one can check that the genus of the corresponding three curves is 4,4 and 10, respectively, so there are only finitely many integral points on these curves.

Family 5. Equation

$$
\left(\begin{array}{c}
x \\
m
\end{array}\right)=y^{n},
$$

where $m \geq 2, n \geq 2, x>m, y \geq 2$ are unknown integers.

For $m=n=2$, equation (5.1) can be written in the form

$$
(2 x-1)^{2}-8 y^{2}=1
$$

which has infinitely many solutions, and all these can be given in a recursive way. For $m=3, n=2$, MEYL [39, $x$ odd] and Watson [57, $x$ even] proved that

$$
\left(\begin{array}{c}
50 \\
3
\end{array}\right)=140^{2}
$$

is the only solution of (5.1).

It was conjectured by Erdős [21] that for $n>2$, equation (5.1) has no solution. ERDős [21] proved this for $n=3$ and for $n \geq 2^{m}$, and OBLÁth [40] for $n=4$ and 5 .

By means of an ingenious elementary method ERDős [22] confirmed his conjecture for $m \geq 4$. For $m<4$, the method of Erdős does not work. 
Using Baker's method, TIJDEMAN [54] proved that for $m=2$ and 3 equation (5.1) has only finitely many solutions, and all of them can be, at least in principle, determined. Later, TERAI [53] showed that for $m=2$ and 3, (5.1) implies $n<4250$.

Finally, GYőRY [25] proved Erdős' conjecture for $m=2,3$ and $n>2$, and hence completed the proof of the following

Theorem 5.1. Apart from the case $(m, n)=(2,2)$, (5.2) gives the only solution of equation (5.1).

Győry's proof combines some results of GYŐRY [24] and DARMON and MEREL [18] on generalized Fermat equations, and a theorem of BENNETT and DE WEGER [5] on binomial Thue equations.

There are several related results in the literature, see e.g. the survey papers [27] and [28] and the references given there. For example, Theorem 5.1 has been extended to the equation

$$
x(x-1) \cdots(x-m+1)=b y^{n}
$$

by SARAdHA [48, $m \geq 4$ ] and GYőRY [26, $m<4$ ], where $b \geq 1$ is also unknown, but has only prime factors not exceeding $m$. For $b=m$ !, the results of [48] and [26] imply Theorem 5.1, while for $b=1$, they give the celebrated theorem of ERDŐS and SELFRIDGE [23] which states that the product of consecutive positive integers is never a power.

Family 6. Equation

$$
\left(\begin{array}{c}
x \\
m
\end{array}\right)=\left(\begin{array}{c}
y \\
n
\end{array}\right),
$$

where $n>m \geq 2$ are fixed integers and $x \geq m, y \geq n$ are unknown integers.

This equation possesses a very extensive literature. There are several scattered computational results for special pairs $(m, n)$. For the resolution of the corresponding equation in the cases $(m, n)=(2,3),(2,4),(2,5),(2,6),(3,4)$ we refer to [1], [19] and [42], [15], [33], [20], respectively. For a nice survey on certain numerical problems and for the cases $(m, n)=(2,8),(3,6),(4,6),(4,8)$ see [52]. Generalizing an earlier result by KIss [36], BRINDZA [12] proved an effective finiteness statement for the solutions to the equation (6.1) with $m=2$. Using some elementary considerations, DE WEGER [20] dealt with equal values of binomial coefficients and proposed the following general conjecture.

Conjecture 6.2. All the solutions of equation (6.1) in positive integers $m$, $n, x, y$ with $n>m \geq 2, x>m, y>n$ are

$$
\left(\begin{array}{c}
16 \\
2
\end{array}\right)=\left(\begin{array}{c}
10 \\
3
\end{array}\right),\left(\begin{array}{c}
56 \\
2
\end{array}\right)=\left(\begin{array}{c}
22 \\
3
\end{array}\right),\left(\begin{array}{c}
153 \\
2
\end{array}\right)=\left(\begin{array}{c}
19 \\
5
\end{array}\right),\left(\begin{array}{c}
221 \\
2
\end{array}\right)=\left(\begin{array}{c}
17 \\
8
\end{array}\right)
$$




$$
\left(\begin{array}{c}
78 \\
2
\end{array}\right)=\left(\begin{array}{c}
15 \\
5
\end{array}\right)=\left(\begin{array}{c}
14 \\
6
\end{array}\right),\left(\begin{array}{c}
21 \\
2
\end{array}\right)=\left(\begin{array}{c}
10 \\
4
\end{array}\right),\left(\begin{array}{c}
120 \\
2
\end{array}\right)=\left(\begin{array}{c}
36 \\
3
\end{array}\right)
$$

and an infinite family

$$
\left(\begin{array}{c}
F_{2 i+2} F_{2 i+3} \\
F_{2 i} F_{2 i+3}
\end{array}\right)=\left(\begin{array}{c}
F_{2 i+2} F_{2 i+3}-1 \\
F_{2 i} F_{2 i+3}+1
\end{array}\right)
$$

for $i=1,2, \ldots$, where $F_{n}$ denotes the $n$th Fibonacci number defined by $F_{0}=0$, $F_{1}=1$ and $F_{n+1}=F_{n}+F_{n-1}$ for $n=1,2, \ldots$.

For general, however, ineffective finiteness results see [7] and [45].

Family 7. Equation

$$
\left(\begin{array}{c}
x \\
m
\end{array}\right)=P_{n}(y)
$$

where $m \geq 2, n \geq 2$ are fixed integers and $x \geq m, y$ are unknown integers.

In the special case $(m, n)=(2,2)$ we have the equation

$$
\left(\begin{array}{l}
x \\
2
\end{array}\right)=2 y^{2}+2 y+1
$$

and a straightforward calculation gives that the transformed equation

$$
(2 x-1)^{2}-(4 y+2)^{2}=5
$$

has no solution in integers $x \geq 2$ and $y$.

For small values of $m$ or $n$ we prove the following

Theorem 7.1. If $m \in\{2,4\}$ and $n \geq 3$ or $n \in\{2,4\}$ and $m \geq 3$ then equation (7.1) implies that $\max (x, y)<c_{4}$, where $c_{4}$ is an effectively computable constant depending only on $n$ or $m$, respectively.

Proof. First suppose that $m \in\{2,4\}$ and $n \geq 3$. We have the equations

and

$$
8 P_{n}(y)+1=(2 x-1)^{2}
$$

$$
24 P_{n}(y)+1=\left(x^{2}-3 x-1\right)^{2},
$$

respectively. Using the fact that

$$
P_{n}(X)=2^{n}\left(\begin{array}{c}
X \\
n
\end{array}\right)+f(X)
$$


where $f(X)$ is an integer-valued polynomial of degree $<n$, and Lemmata 1 and 2 give our statement for $n \geq 6$. If $n=3,4,5$ then an easy calculation shows that the corresponding polynomials have at least three simple zeros, and the proof is completed in these cases as well

Now assume that $n \in\{2,4\}$ and $m \geq 3$. We get the equations

$$
2\left(\begin{array}{c}
x \\
m
\end{array}\right)-1=(2 y+1)^{2}
$$

and

$$
3\left(\begin{array}{c}
x \\
m
\end{array}\right)+5=2\left(y^{2}+y+2\right)^{2},
$$

respectively. Our Lemmata 5 and 2 completes the proof for $m \geq 3$.

Theorem 7.2. Suppose that $\min \{m, n\} \geq 3$. Then (7.1) has only finitely many solutions in integers $x$ and $y$.

Proof. On supposing the contrary and using Lemma 7 we have

$$
\left(\begin{array}{c}
a X+b \\
m
\end{array}\right)=\phi(f(X))
$$

and

$$
P_{n}(\tilde{a} X+\tilde{b})=\phi(g(X)),
$$

where $(f, g)$ is a standard pair, $\phi(X) \in \mathbb{Q}[X]$ and $a, b, \tilde{a}, \tilde{b} \in \mathbb{Q}$ with $a \tilde{a} \neq 0$. We will prove that $k:=\operatorname{deg} \phi=1$. Indeed, it is clear that the ratio of the leading coefficients of the polynomials $\left(\begin{array}{c}a X+b \\ m\end{array}\right)$ and $P_{n}(\tilde{a} X+\tilde{b})$ is a $k$ th power in $\mathbb{Q}$. On the other hand, this ratio is

$$
\frac{a^{m} \cdot n !}{2^{n} \cdot \tilde{a}^{n} \cdot m !} .
$$

Since $m=k \cdot \operatorname{deg} f$ and $n=k \cdot \operatorname{deg} g$ are divisible by $k$, then the number $n ! / m$ ! is a $k$ th power in $\mathbb{Q}$. Lemma 8 gives that $k=1$ or $k \geq 2,|n-m|=1$. However, in the second case, $2 \leq k \leq \operatorname{gcd}(m, n)=1$ and we have a contradiction Thus we obtain

$$
\left(\begin{array}{c}
a X+b \\
m
\end{array}\right)=e_{1} f(X)+e_{0}
$$

and

$$
P_{n}(\tilde{a} X+\tilde{b})=f_{1} g(X)+f_{0},
$$

where $e_{0}, e_{1}, f_{0}, f_{1}$ are rational numbers with $e_{1} f_{1} \neq 0$. By the condition $\min \{m, n\} \geq 3,(f, g)$ is not a standard pair of the second kind, further by Theorem 7.1, we get that $(f, g)$ is not a standard pair of the fifth kind. Using Lemma 9 
and Theorem 7.1 our theorem is proved apart from the case $(m, n)=(3,3)$. In this case the corresponding curve is

$$
\frac{x(x-1)(x-2)}{6}-\frac{4}{3} y^{3}-2 y^{2}-\frac{8}{3} y-1=0,
$$

its genus determined by MAPLE is one, so we have only finitely many integer solutions.

Family 8. Equation

$$
P_{m}(x)=y^{n},
$$

where $m \geq 2$ is fixed and $x, y, n \geq 2$ are unknown positive integers with $(m, n) \neq$ $(2,2)$.

In the trivial case $(m, n)=(2,2)$ we have $P_{2}(x)=2 x^{2}+2 x+1$ so the corresponding diophantine equation is

or equivalently,

$$
2 x^{2}+2 x+1=y^{2},
$$

$$
(2 x+1)^{2}-2 y^{2}=-1
$$

which is a Pellian equation with infinitely many solutions. We can rewrite the polynomial $P_{n}(X)$ as

$$
P_{n}(X)=\sum_{j=0}^{n}\left(\begin{array}{c}
n \\
j
\end{array}\right)\left(\begin{array}{c}
X+n-j \\
n
\end{array}\right)=2^{n}\left(\begin{array}{l}
X \\
n
\end{array}\right)+f(X),
$$

where $f(X)$ is an integer-valued polynomial of degree $<n$. So from Lemma 1 we get that $P_{n}(X)$ has at least three simple zeros for $n \geq 6$. In the remaining cases we obtain

$$
\begin{aligned}
& P_{2}(X)=2 X^{2}+2 X+1, P_{3}(X)=\frac{4}{3} X^{3}+2 X^{2}+\frac{8}{3} X+1, \\
& P_{4}(X)=\frac{2}{3} X^{4}+\frac{4}{3} X^{3}+\frac{10}{3} X^{2}+\frac{8}{3} X+1,
\end{aligned}
$$

and

$$
P_{5}(X)=\frac{4}{15} X^{5}+\frac{2}{3} X^{4}+\frac{8}{3} X^{3}+\frac{13}{3} X^{2}+\frac{46}{15} X+1,
$$

and one can calculate their non-zero discriminants showing that these polynomials possess only simple zeros. Thus the following statement follows from Lemmata 2 and 3 . 
Theorem 8.1. Let $m, n$ be integers with $m \geq 2, n \geq 2$ and suppose that $(m, n) \neq(2,2)$. The equation (8.1) in integers $x, y$ and $n$ implies $\max \{|x|,|y|, n\}<C$ where $C$ is an effectively computable constant depending only on $m$.

We note that Theorems 8.1 and 8.2 below are new.

CoHn [17] resolved the equation $x^{2}+1=y^{n}$ and proved that all the solutions of this equation in integers $x, y, n$ with $n>1$ are $x=y=1$ and $x=239, y=13$, $n=4$. Using this result we have

Theorem 8.2. All the solutions of the equation $P_{2}(x)=y^{n}$ in integers $x, y$ and $n>2$ are $x=0, y=1$ and $x=119, y=13, n=4$.

Family 9. Equation

$$
P_{m}(x)=P_{n}(y)
$$

where $n>m \geq 2$ are fixed integers and $x, y$ are unknown integers.

Hajdu studied the equation (9.1) for small values of $m$ and $n$ and resolved the corresponding elliptic type diophantine equations, see [31] and [32]. Further, he conjectured that the equation has only finitely many solutions for $n>m=2$. This conjecture was confirmed by Kirschenhofer, Pethő and Tichy [35]. Later, using the Bilu-Tichy Theorem, Bilu, Stoll and Tichy [9] extended their result to the general case by proving an ineffective finiteness statement for the number of solutions $x$ and $y$ for every pair $(m, n)$.

ACKNOWLEDGEMENTs. The authors are grateful to the referee for the careful reading of the manuscript and for her/his helpful remarks.

\section{References}

[1] È. T. Avanesov, Solution of a problem on figurate numbers, Acta Arith. 12 (1966/1967), 409-420.

[2] È. T. Avanesov, The Diophantine equation $3 y(y+1)=x(x+1)(2 x+1)$, Volž. Mat. Sb. 8 (1971), 3-6.

[3] A. BAKer, Bounds for the solutions of the hyperelliptic equation, Proc. Cambridge Philos. Soc. 65 (1969), 439-444.

[4] M. BeCK and S. Robins, Computing the continuous discretely, Undergraduate Texts in Mathematics, Springer, New York, 2007.

[5] M. A. Bennett and B. M. M. De Weger, On the Diophantine equation $\left|a x^{n}-b y^{n}\right|=1$, Math. Comp. 67 (1998), 413-438.

[6] M. A. Bennett, K. Győry and Á. Pintér, On the Diophantine equation $1^{k}+2^{k}+\ldots$ $+x^{k}=y^{n}$, Compos. Math. 140 (2004), 1417-1431. 
[7] F. Beukers, T. N. Shorey and R. Tijdeman, Irreducibility of polynomials and arithmetic progressions with equal products of terms, Number Theory in Progress, Vol. 1 (Zakopane-Kościelisko, 1997), de Gruyter, Berlin, 1999.

[8] Y. F. Bilu, B. Brindza, P. Kirschenhofer, Á. Pintér and R. F. Tichy, Diophantine equations and Bernoulli polynomials. With an appendix by A. Schinzel, Compositio Math. 131 (2002), 173-188.

[9] Y. F. Bilu, T. Stoll, and R. F. Tichy, Octahedrons with equally many lattice points, Period. Math. Hungar. 40 (2000), 229-238.

[10] Y. F. Bilu and R. F. Tichy, The Diophantine equation $f(x)=g(y)$, Acta Arith. 95 (2000), 261-288.

[11] B. BRINDZA, On some generalizations of the Diophantine equation $1^{k}+2^{k}+\cdots+x^{k}=y^{z}$, Acta Arith. 44 (1984), 99-107.

[12] B. Brindza, On a special superelliptic equation, Publ. Math. Debrecen 39 (1991), 159-162.

[13] B. Brindza and Á. Pintér, On equal values of power sums, Acta Arith. 77 (1996), 97-101.

[14] B. Brindza and Á. Pintér, On the number of solutions of the equation $1^{k}+2^{k}+\cdots$ $+(x-1)^{k}=y^{z}$, Publ. Math. Debrecen 56 (2000), 271-277.

[15] Y. Bugeaud, M. Mignotte, S. Siksek, M. Stoll and Sz. Tengely, Integral points on hyperelliptic curves, Algebra Number Theory 2 (2008), 859-885.

[16] J. W. S. CASSELS, Integral points on certain elliptic curves, Proc. London Math. Soc. 14a (1965), 55-57.

[17] J. H. E. Cohn, Perfect Pell powers, Glasgow Math. J. 38 (1996), 19-20.

[18] H. DARmon and L. Merel, Winding quotients and some variants of Fermat's last theorem, J. Reine Angew. Math. 490 (1997), 81-100. ,

[19] B. M. M. DE Weger, A binomial Diophantine equation, Quart. J. Math. Oxford Ser. (2) 47 (1996), 221-231.

[20] B. M. M. DE WeGer, Equal binomial coefficients: some elementary considerations, $J$. Number Theory 63 (1997), 373-386.

[21] P. ERdős, Note on the product of consecutive integers (II), J. London Math. Soc. 14 (1939), 245-249.

[22] P. Erdős, On a Diophantine equation, J. London Math. Soc. 26 (1951), 176-178.

[23] P. ERdős and J. L. Selfridge, The product of consecutive integers is never a power, Illinois J. Math. 19 (1975), 292-301.

[24] K. GYőRY, Über die diophantische Gleichung $x^{p}+y^{p}=c z^{p}$, Publ. Math. Debrecen 13 (1966), 301-305.

[25] K. GYőRY, On the Diophantine equation $\left(\begin{array}{l}n \\ k\end{array}\right)=x^{l}$, Acta Arith. 80 (1997), 289-295.

[26] K. GYőRY, On the Diophantine equation $n(n+1) \cdots(n+k-1)=b x^{l}$, Acta Arith. 83 (1998), 87-92.

[27] K. GYŐRY, Perfect powers in products with consecutive terms from arithmetic progressions, More Sets, Graphs and Nnumbers, Bolyai Soc. Math. Stud. Vol. 15, Springer, Berlin, 2006, $143-155$.

[28] K. GYőRY, Perfect powers in products with consecutive terms from arithmetic progressions, II, Erdős Centennial, Bolyai Soc. Math. Stud., Springer, Berlin, 2013, 311-324.

[29] K. GYőRY and Á PINTÉR, On the equation $1^{k}+2^{k}+\cdots+x^{k}=y^{n}$, Publ. Math. Debrecen 62 (2003), 403-414.

[30] K. Györy, R. Tijdeman and M. Voorhoeve, On the equation $1^{k}+2^{k}+\cdots+x^{k}=y^{z}$, Acta Arith. 37 (1980), 233-240. 
[31] L. HAJDU, On a Diophantine equation concerning the number of integer points in special domains II, Publ. Math. Debrecen 51 (1997), 331-342.

[32] L. HAJDU, On a Diophantine equation concerning the number of integer points in special domains, Acta Math. Hungar. 78 (1998), 59-70.

[33] L. Hajdu, and Á. Pintér, Combinatorial Diophantine equations, Publ. Math. Debrecen 56 (2000), 391-403.

[34] JR., M. J. JACOBSON, Á PintÉR, and P. G. WALSh, A computational approach for solving $y^{2}=1^{k}+2^{k}+\cdots+x^{k}$, Math. Comp. 72 (2003), 2099-2110 (electronic).

[35] P. Kirschenhofer, A. Pethő and R. F. Tichy, On analytical and Diophantine properties of a family of counting polynomials, Acta Sci. Math. (Szeged) 65 (1999), 47-59.

[36] P. KIss, On the number of solutions of the Diophantine equation $\left(\begin{array}{l}x \\ p\end{array}\right)=\left(\begin{array}{l}y \\ 2\end{array}\right)$, Fibonacci Quart. 26 (1988), 127-130.

[37] T. KovÁcs, Combinatorial Diophantine equations - the genus 1 case, Publ. Math. Debrecen 72 (2008), 243-255.

[38] W. Ljungaren, Solution complète de quelques équations du sixième degré à deux indéterminées, Arch. Math. Naturvid. 48 (1946), 35.

[39] A. J. J. MeYL, Question 1194, Nouv, Ann. Math 2 (1878), 464-467.

[40] R. ОвцÁth, Note on the binomial coefficients, J. London Math. Soc. 23 (1948), 252-253.

[41] Á. PintÉr, On the number of simple zeros of certain polynomials, Publ. Math. Debrecen 42 (1993), 329-332.

[42] Á. Pintér, A note on the Diophantine equation $\left(\begin{array}{l}x \\ 4\end{array}\right)=\left(\begin{array}{l}y \\ 2\end{array}\right)$, Publ. Math. Debrecen 47 (1995), 411-415.

[43] Á. Pintér, A note on the equation $1^{k}+2^{k}+\cdots+(x-1)^{k}=y^{m}$, Indag. Math. (N.S.) 8 (1997), 119-123.

[44] Á. PinTÉR, On a class of Diophantine equations related to the numbers of cells in hyperplane arrangements, J. Number Theory 129 (2009), 1664-1668.

[45] Cs. Rakaczki, On the Diophantine equation $\left.F\left(\begin{array}{l}x \\ n\end{array}\right)\right)=b\left(\begin{array}{l}y \\ m\end{array}\right)$, Period. Math. Hungar. 49 (2004), 119-132.

[46] Cs. Rakaczki, On the Diophantine equation $S_{m}(x)=g(y)$, Publ. Math. Debrecen 65 (2004), 439-460.

[47] Cs. RAKaCzKI, On some generalizations of the Diophantine equation $s\left(1^{k}+2^{k}+\cdots+x^{k}\right)+$ $r=d y^{n}$, Acta Arith. 151 (2012), 201-216.

[48] N. SARAdHA, On perfect powers in products with terms from arithmetic progressions, Acta Arith. 82 (1997), 147-172.

[49] J. J. SchäfFER, The equation $1^{p}+2^{p}+3^{p}+\cdots+n^{p}=m^{q}$, Acta Math. 95 (1956), 155-189.

[50] J. J. Schäffer and R. Tijdeman, On the equation $y^{m}=P(x)$, Acta Arith. (1976), 199-204.

[51] T. Stoll and R. F. Tiснy, Diophantine equations involving general Meixner and Krawtchouk polynomials, Quaestiones Mathematicae 28, 105-115.

[52] R. J. Stroeker and B. M. M. De Weger, Elliptic binomial Diophantine equations, Math. Comp. 68 (1999), 1257-1281.

[53] N. TeraI, On a Diophantine equation of Erdős, Proc. Japan Acad. Ser. A Math. Sci. 70 (1994), 213-217.

[54] R. Tijdeman, Applications of the Gel'fond-Baker method to rational number theory, Topics in Number Theory, (Proc. Colloq., Debrecen, 1974), Colloq. Math. Soc. János Bolyai, Vol. 13, North-Holland, Amsterdam, 1976. 
[55] S. UсhiYama, Solution of a Diophantine problem, Tsukuba J. Math. 8 (1984), 131-157.

[56] M. Voorhoeve, K. Győry and R. Tijdeman, On the Diophantine equation $1^{k}+2^{k}+\cdots$ $+x^{k}+R(x)=y^{z}$, Acta Math. 143 (1979), 1-8.

[57] G. N. Watson, The problem of the square pyramid, Messenger of Math 48 (1918), 1-22.

[58] P. Z. Yuan, On a special Diophantine equation $a\left(\begin{array}{l}x \\ n\end{array}\right)=b y^{r}+c$, Publ. Math. Debrecen 44 (1994), 137-143.

KÁLMÁN GYÖRY

INSTITUTE OF MATHEMATICS

UNIVERSITY OF DEBRECEN

H-4010 DEBRECEN, P.O. BOX 12

HUNGARY

E-mail: gyory@science.unideb.hu

GYÖNGYVÉR PÉTER

INSTITUTE OF MATHEMATICS

UNIVERSITY OF DEBRECEN

H-4010 DEBRECEN, P.O. BOX 12

HUNGARY

E-mail: pgyongyver@gmail.com

\author{
TÜNDE KOVÁCS \\ INSTITUTE OF MATHEMATICS \\ UNIVERSITY OF DEBRECEN \\ H-4010 DEBRECEN, P.O. BOX 12 \\ HUNGARY \\ E-mail: tkovacs@science.unideb.hu \\ ÁKOS PINTÉR \\ INSTITUTE OF MATHEMATICS \\ MTA-DE RESEARCH GROUP \\ "EQUATIONS FUNCTIONS AND CURVES" \\ HUNGARIAN ACADEMY OF SCIENCES \\ AND UNIVERSITY OF DEBRECEN \\ H-4010 DEBRECEN, P.O. BOX 12 \\ HUNGARY \\ E-mail: apinter@science.unideb.hu
}

(Received August 28, 2013; revised November 6, 2013) 